\title{
The effect of orthostatic hypotension on cerebral blood flow and middle cerebral artery velocity in autonomic failure, with observations on the action of ephedrine
}

\author{
D J BROOKS, S REDMOND, C J MATHIAS, R BANNISTER, L SYMON \\ From the National Hospital for Nervous Diseases, Queen Square, London
}

SUMMARY Cerebral blood flow (CBF) and middle cerebral artery velocity (MCAv) have been measured using ${ }^{133}$ xenon washout and transcranial Doppler in ten patients with autonomic failure. Four pure autonomic failure and four multiple system atrophy patients behaved similarly: tilting them sufficiently to induce significant orthostatic hypotension without causing syncopal symptoms led to a significant fall in their mean MCAv, but no change in their mean CBF. These findings suggest that cerebral autoregulation is preserved in autonomic failure, orthostatic hypotension resulting in a reactive vasodilatation which lowers MCAv, reduces vascular resistance, and maintains CBF. Ephedrine helped to correct the orthostatic hypotension, but had no direct effect on CBF. Two siblings with orthostatic hypotension secondary to dopamine- $\beta$-hydroxylase deficiency also had preserved cerebral autoregulation, but ephedrine led to paradoxical hypotension in these patients.

The brain autoregulates, that is over a range of cerebral perfusion pressures from $70-140 \mathrm{~mm} \mathrm{Hg}$ the brain is able to maintain a constant level of blood flow. ${ }^{1-3}$ The mechanism of autoregulation is obscure, but probably involves myogenic reflexes acting at an arteriolar level. ${ }^{4}$ Whether the autonomic nervous system plays a significant role in controlling CBF is unclear. The extracranial circulation is richly innervated, and animal studies have shown that stimulation of the sympathetic and parasympathetic supplies to this circulation results in vasoconstriction and vasodilatation of pial arteries respectively. ${ }^{5-7}$ The smaller, penetrating, cerebral arteries, however, have a sparse autonomic supply, ${ }^{8}$ and it is likely that they are less affected by autonomic activity. Consequently one might predict that control of CBF will be largely independent of the autonomic supply. Transection of the autonomic supply in animals does not appear to impair their cerebral autoregulation, and leads to little change in $\mathrm{CBF} .^{9-12}$ Stimulation of the sympathetic

Address for reprint requests: Dr D J Brooks, MRC Cyclotron Unit, Hammersmith Hospital, Du Cane Road, London W12 0HS, UK.

Received 23 December 1988.

Accepted 3 March 1989 supply to the extracranial circulation also results in only small reductions in $\mathrm{CBF} .^{-1224}$ Intracarotid infusions of noradrenaline have little effect on CBF in man, ${ }^{13}$ while internal carotid artery strips show little contractility when exposed to physiological levels of noradrenaline ${ }^{14}$ The above findings all argue against the sympathetic supply playing a significant role in the control of CBF.

There have been several studies on the effects of autonomic failure on cerebral autoregulation in man Caronna and Plum, ${ }^{15}$ computing CBF changes in directly from arterio-venous oxygen differences reported intact autoregulation in three subjects with multiple system atrophy (MSA), but impaired autoregulation in a patient with pure autonomic failure (PAF). Meyer $e t$ al,$^{16}$ also using arterio-venous oxygen differences to measure CBF changes, found impaired autoregulation in all three of their MSA subjects. Nanda et al, ${ }^{17}$ and Thomas and Bannister, ${ }^{18}$ using ${ }^{133}$ xenon washout to measure CBF, both found intact cerebral autoregulation in their groups of PAF and MSA subjects.

In view of the contradictory findings previously reported, we have re-examined the effect of autonomic failure on cerebral autoregulation in man. We have also examined the effect of orthostatic hypotension 962 
on middle cerebral artery velocity (MCAv). This parameter reflects vessel calibre, being elevated in artery spasm secondary to subarachnoid haemorrhage,$^{19}$ and reduced in arterial dilatation secondary to carotid artery occlusion. ${ }^{20}$ Such MCAv measurements provide an indirect means of examining the effect of orthostatic hypotension on cerebrovascular resistance in autonomic failure. Ephedrine is a rapidly acting oral sympathomimetic agent which is often used to correct orthostatic hypotension. ${ }^{2125}$ We have examined the effect of ephedrine on cerebral haemodynamics in autonomic failure (AF) to see whether this agent has a direct beneficial effect on CBF, or simply acts as a hypertensive agent.

\section{Methods}

Four patients with autonomic failure in association with multiple system atrophy, four patients with pure autonomic failure, and a brother and sister with orthostatic hypotension in association with autosomal recessively inherited dopamine- $\beta$-hydroxylase deficiency and undetectable plasma catecholamine levels, were studied. Patient details are listed in table 1 . No patient had evidence of a peripheral neuropathy clinically, or on EMG. Patients were studied both supine, and tilted head-up to a degree that led to a significant fall in blood pressure (BP) without causing syncopal symptoms. Tilted measurements were then repeated one hour after a $30 \mathrm{mg}$ oral dose of ephedrine. Heart rate and mean arterial BP were recorded at 1-2 minute intervals throughout the study using a Centron Blood Pressure Analyser.

Cerebral blood flow was measured using the intravenous ${ }^{133}$ xenon washout technique. ${ }^{23} 6-8 \mathrm{mCi}$ of ${ }^{133} \mathrm{Xe}$ in saline (Amersham, UK) were injected as an intravenous bolus. Cerebral hemisphere washout curves were recorded with a Novo Cerebrograph 10a, a detector being positioned over each temporo-parietal area. End-expiratory ${ }^{133} \mathrm{Xe}$ was also

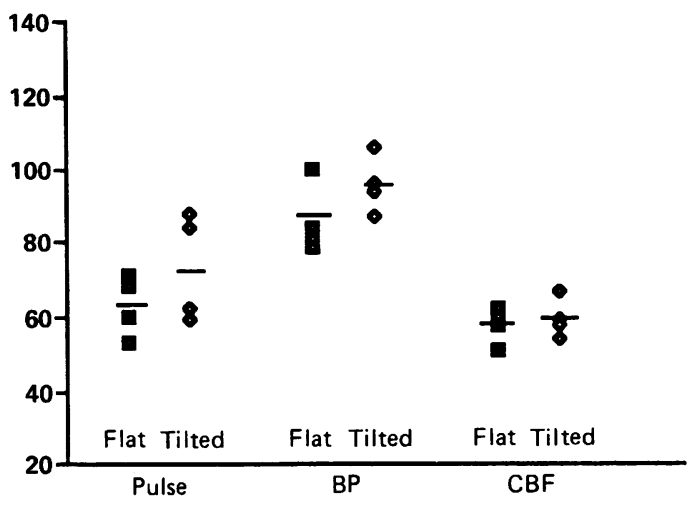

Fig 1 The effect of tilt on pulse, BP, and CBF in normal subjects.

measured, and whole hemisphere CBF was calculated using the initial slope index approach. ${ }^{26}$ Middle cerebral artery velocity was measured using an EME TC $2642 \mathrm{MHz}$ pulsed transcranial Doppler velocimeter placed over the temporal area. ${ }^{20}$

\section{Results}

Figure 1 shows the effect of $45^{\circ}$ head-up tilt on the heart rate, mean BP, and CBF of four normal subjects aged 28-38 years. Mean heart rate increased by $16 \%$, BP by $12 \%$, and there was no significant change in CBF. MCAv values were also unaffected by tilting. Figure 2 shows the effect of head-up tilt on the combined group of eight MSA and PAF patients. Their mean heart rate increased by $5 \%$ while their mean BP fell by $20 \%$. There was negligible change in their mean CBF but their mean MCAv fell significan-

Table 1 Clinical details of patients with autonomic failure

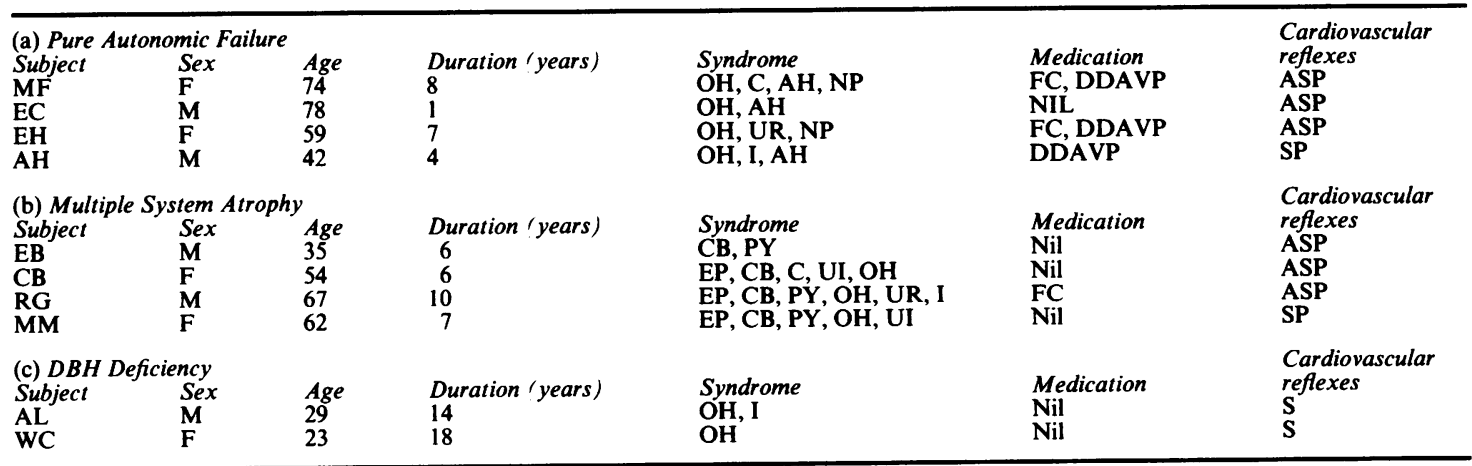

Abbreviations: $\mathrm{OH}-$ Orthostatic hypertension, $\mathrm{C}$-constipation, $\mathrm{AH}$-anhidrosis, NP-nocturnal polyuria, UR-urinary retention, UIurinary incontinence, I-impotence, EP-extrapyramidal rigidity, CB-cerebellar ataxia, PY-pyramidal dysfunction, ASP-afferent, sympathetic, parasympathetic lesions, DDAVP-Desmopressin, FC-Fludrocortisone. 


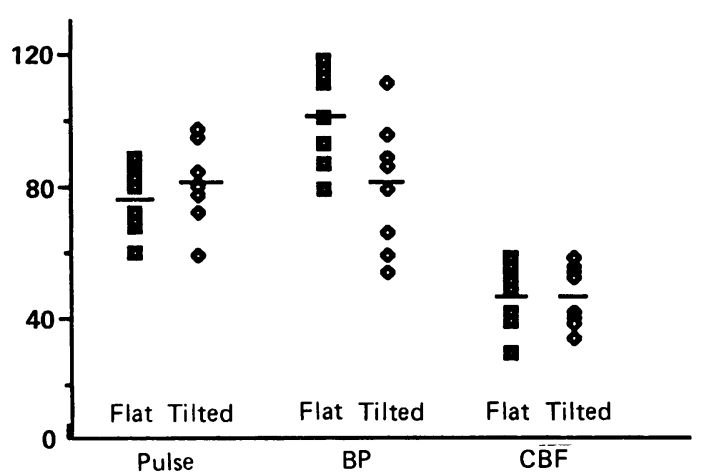

Fig 2 The effect of tilt on pulse, $B P$, and $C B F$ in autonomic failure.

tly by $16 \%$. These results are summarised in table 2 . Figure 3 shows the effect of progressive tilt on one subject with PAF. It can be seen that MCAv falls in parallel with mean arterial $\mathrm{BP}, \mathrm{CBF}$ remaining essentially constant.

The effect of ephedrine on the haemodynamics of the tilted MSA and PAF patients is detailed in table 3. Ephedrine resulted in a significant increase in mean heart rate and BP, a smaller increase in mean MCAv, but no significant change in CBF. Figure 4 shows the effect of ephedrine on the brother and sister with dopamine- $\beta$-hydroxylase deficiency, and undetectable plasma catecholamine levels. Ephedrine produced an increase in their supine heart rate, but a fall in their supine BP. No significant changes in their CBF were observed.

\section{Discussion}

Our findings demonstrate that cerebral autoregulation is preserved in autonomic failure. In our group of eight patients with PAF and MSA, a fall of $20 \mathrm{~mm} \mathrm{Hg}$ in mean arterial BP led to no significant change in mean CBF. There was no difference in behaviour between the PAF and MSA subjects, both sets of patients maintaining their $\mathrm{CBF}$ when tilted. Our findings are in

Table 2 The effect of head-up tilt on cerebral haemodynamics in autonomic failure

\begin{tabular}{llllll}
\hline & \multicolumn{2}{l}{ Controls (4) } & & \multicolumn{2}{l}{$M S A / P A F(8)$} \\
\cline { 2 - 3 } \cline { 5 - 6 } \cline { 5 - 6 } & supine & tilted & & supine & tilted \\
\hline Heart rate & $63(8)$ & $73(15)$ & & $76(10)$ & $81(12)$ \\
Mean BP $(\mathrm{mmHg})$ & $86(10)$ & $96(8)^{*}$ & & $102(15)$ & $80(19) \dagger$ \\
CBF $(\mathrm{ml} / 100 \mathrm{~g} / \mathrm{min})$ & $58(5)$ & $60(5)$ & & $47(10)$ & $47(10)$ \\
MCAv $\left(\mathrm{cm} \mathrm{s}^{-1}\right)$ & $51(6)$ & $51(6)$ & & $56(13)$ & $47(14)^{*}$ \\
\hline
\end{tabular}

${ }^{*} p<0.01,+p<0.005,()=$ Standard Deviation. (Student's paired $\mathrm{t}$ test)

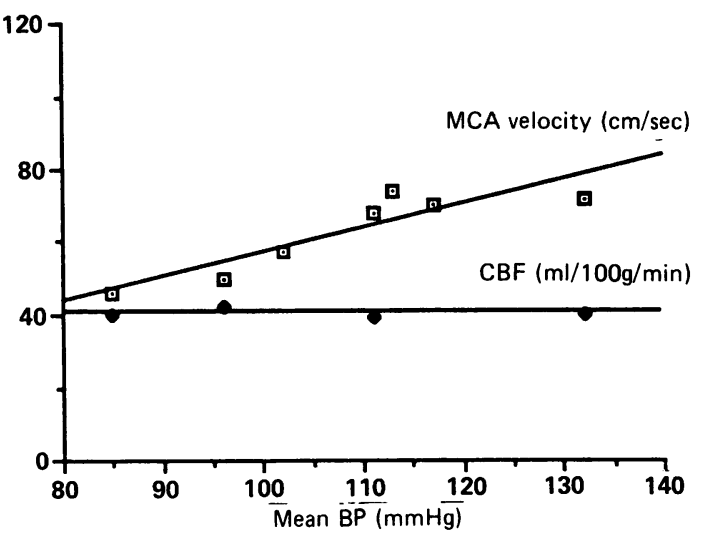

Fig $3 E H M C A$ velocity and $C B F$ vs $B P$.

agreement with Nanda et al, ${ }^{17}$ and Thomas and Bannister, ${ }^{18}$ whose PAF and MSA subjects also showed preserved cerebral autoregulation. These sets of workers, like us, used the ${ }^{133}$ xenon washout technique to measure $\mathrm{CBF}$.

In contrast to our findings Meyer et al ${ }^{16}$ found impaired cerebral autoregulation in their three MSA subjects. These workers used arterio-venous oxygen difference measurements to assess CBF changes. The is an indirect technique which assumes constant levels of cerebral oxygen utilisation when patients are tilted both head-up and head-down. Consequently artefae tual swings in CBF are liable to result using the technique. One of these workers' three patients was tilted such that his mean arterial BP fell to $32 \mathrm{~mm} \mathrm{Hg}$, well below the autoregulatory range. As such it is not surprising that this particular subject showed a significant fall in CBF with BP.

Caronna and Plum, ${ }^{15}$ like Meyer et al ${ }^{16}$ used the arteriovenous oxygen difference technique to monitor $\overrightarrow{\vec{B}}$ CBF changes in three MSA and a PAF subject. Unlike Meyer et $a l,{ }^{16}$ these workers found intact cerebral autoregulation in their three MSA subjects. Their PAF subject, however, showed a clear dependence of CBF on mean arterial BP. Caronna and Plum ${ }^{15}$ suggested that as PAF subjects have an increased

Table 3 The effect of ephedrine on cerebral haemodynamics in tilted MSA and PAF patients

\begin{tabular}{|c|c|c|}
\hline & Baseline & Treated \\
\hline $\begin{array}{l}\text { Heart rate } \\
\text { Mean BP }(\mathrm{mmHg}) \\
\text { CBF }(\mathrm{ml} / 100 \mathrm{~g} / \mathrm{min}) \\
\text { MCAv }\left(\mathrm{cm} \mathrm{s}^{-1}\right)\end{array}$ & $\begin{array}{l}81(12) \\
80(19) \\
47(10) \\
47(14)\end{array}$ & $\begin{array}{l}91(11)^{*} \\
92(17)^{*} \\
49(11) \\
51(10)^{*}\end{array}$ \\
\hline
\end{tabular}

${ }^{*} p<0.05,()=$ Standard Deviation.

(Student's paired $t$ test). 

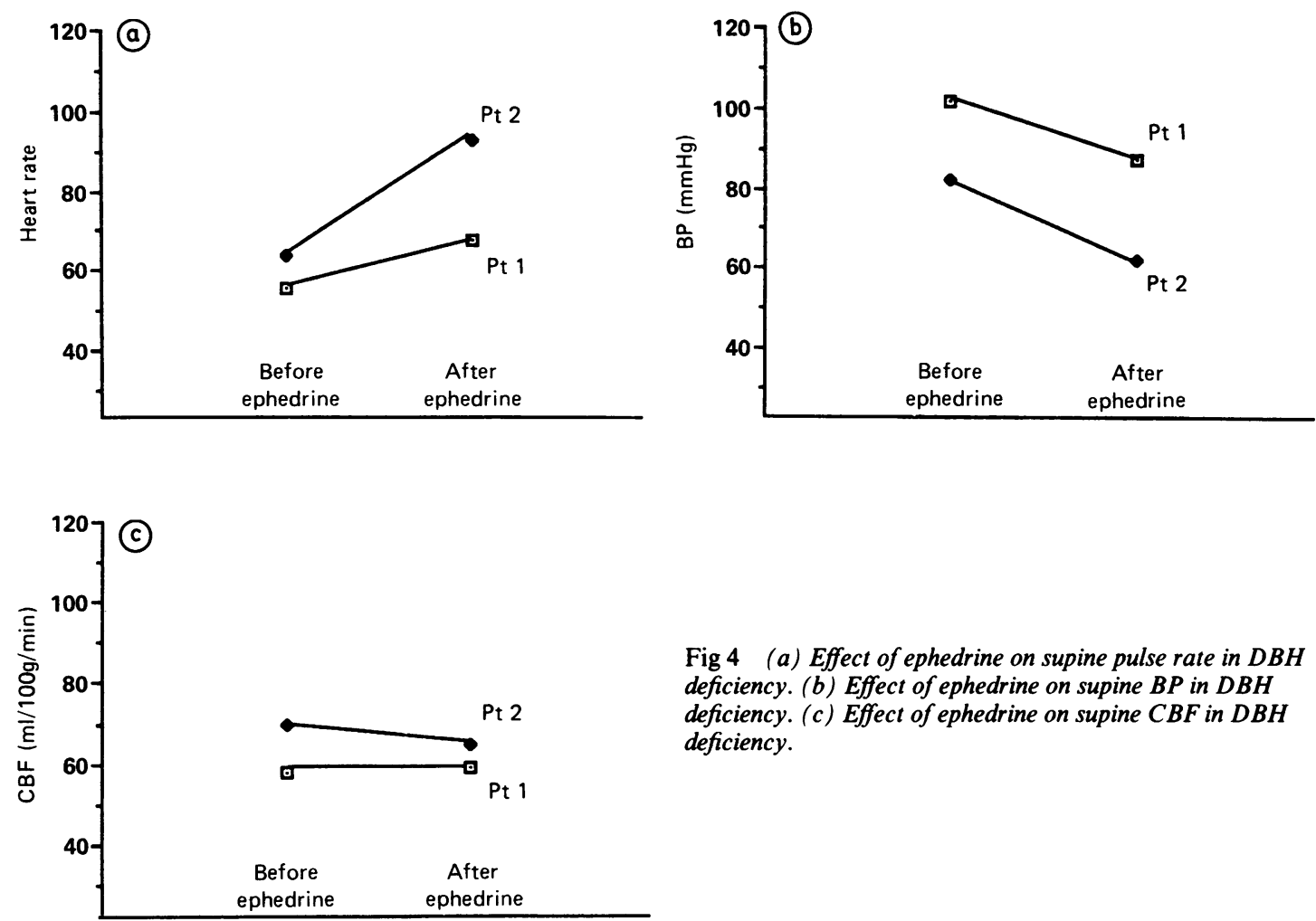

Fig 4 (a) Effect of ephedrine on supine pulse rate in $D B H$ deficiency. (b) Effect of ephedrine on supine BP in DBH deficiency. (c) Effect of ephedrine on supine CBF in DBH deficiency.

destruction of post-ganglionic neurons in their autonomic nervous system compared to MSA subjects, PAF patients would be more likely to show impaired cerebral autoregulation. This, however is not our experience, or that of Nanda et $a l^{17}$ and Thomas and Bannister, ${ }^{18}$ both of whose PAF subjects autoregulated normally. Currently we have no explanation for the failure of Caronna and Plum's ${ }^{15}$ PAF subject to autoregulate.

Our finding of intact autoregulation in autonomic failure patients supports the concept that CBF is controlled by myogenic reflexes at an arteriolar level. ${ }^{4}$ Arterioles are poorly innervated by the autonomic nervous system, ${ }^{8}$ and animal studies have shown insignificant changes in CBF when the cerebral autonomic supply is stimulated or transected in animals. $^{91224}$ Obliteration of the cerebral autonomic supply does not appear to affect autoregulation.9 What then is the role of the autonomic nervous system in controlling CBF, and why are extracranial, but not intracranial vessels richly innervated? ${ }^{8}$ Harper et al ${ }^{10}$ have suggested that the extra- and intra-parenchymal vessels act in series, changes in extra parenchymal resistance being counter-balanced by the intraparen- chymal circulation. In this way the intracranial circulation can vasodilate to maintain $\mathrm{CBF}$ when extracranial vasospasm occurs secondary to subarachnoid haemorrhage or acute hypotension, while the extracranial circulation protects arterioles against the effects of acute hypertension due to sympathetic overactivity.

Our patients with autonomic failure all showed a parallel fall in MCAv with their orthostatic hypotension. MCAv essentially reflects middle cerebral artery calibre, being elevated in the presence of MCA spasm, and reduced when MCAs are vasodilated. ${ }^{1920} \mathrm{~A}$ fall in MCAv with BP suggests that the extracranial circulation is vasodilating, and so reducing cerebrovascular resistance when patients with autonomic failure become hypotensive. Such vasodilatation of the extracranial circulation in the face of hypotension is likely to be a normal, rather than pathological, protective mechanism. Four of our control subjects showed reduced MCAvs when hypotensive during Stage II of a Valsalva manoeuvre, MCAv recovering within seconds of releasing their increased intraabdominal pressure (Brooks DJ, unpublished observations). Tilting normal subjects increases their 
BP slightly, and has no significant effect on their MCAv.

Ephedrine acts mainly as an indirect sympathomimetic agent, with weaker direct agonist activity. ${ }^{2225}$ It is frequently used as a hypertensive agent in orthostatic hypotension, acting within 20-30 minutes when taken orally provided normal gastrointestinal motility is present. It can be seen that in our PAF and MSA subjects ephedrine acted to increase heart rate, BP, and to a lesser extent MCAv. There was no significant change in CBF. This suggests that ephedrine has no direct beneficial action on CBF, acting as a positive chronotropic agent and vasoconstrictor. Its use in autonomic failure lies in its ability to maintain standing patients in the autoregulatory BP range. Unfortunately supine hypertension is frequently a problem with this agent. ${ }^{25}$

Dopamine- $\beta$-hydroxylase deficiency is a rate autonomal recessive condition in which the enzyme for converting dopamine to noradrenaline is absent. ${ }^{27}$ Patients have undetectable plasma noradrenaline and adrenaline, and raised plasma dopamine levels. They present with orthostatic hypotension and impaired ejaculation. ${ }^{27}$ Our brother and sister with DBH deficiency showed a paradoxical reaction to ephedrine, their supine BP falling after treatment. Their CBF was unchanged however, demonstrating that cerebral autoregulation remains intact in this condition. The mechanism of the ephedrine-induced hypotension in DBH deficiency remains unclear. One possibility is that a direct agonist effect of ephedrine on vasodilatory $\beta$-adrenoreceptors occurs.

\section{Conclusions}

Our findings can be summarised as follows:

(a) Cerebral autoregulation is preserved in pure autonomic failure, and in autonomic failure secondary to multiple system atrophy and dopamine- $\beta$-hydroxylase deficiency.

(b) Orthostatic hypotension results in a reactive vasodilatation in autonomic failure patients, cerebral blood flow being maintained by lowering vascular resistance.

(c) Ephedrine acts as a hypertensive agent in PAF and MSA subjects, having no direct effect on CBF. In dopamine- $\beta$-hydroxylase deficiency, however, ephedrine results in paradoxical hypotension.

\section{References}

1 Gottstein U. Physiologie and Pathophysiologie des Hirnkreislaufs. Med Welt (Stuttg.) 1965;15:715-26.

2 Harper AM. Autoregulation of cerebral blood flow-Influence of the arterial blood pressure on the blood flow through the cerebral cortex. J Neurol Neurosurg Psychiatry 1975;29: 398-403.

3 Lassen NA. Control of cerebral circulation in health and disease. Circulat Res 1974;34:749-60.
4 Strandgaard S, Paulson OB. Cerebral Autoregulation. Stroke 1984;15:413-6.

5 Forbes HS, Cobb S. Vasomotor control of cerebral vessels. Brain 1938;61:221-33.

6 Forbes HS, Schmidt CF, Nason GI. Evidence of vasodilator innervation in the parietal cortex of the cat. Am J Physiol 1939;125:216-9.

7 Chorobski J, Penfield W. Cerebral vasodilator nerves and their pathway from the medulla oblongata. With observations on the pial and intracerebral vascular plexus. Arch Neurol Psychiat 1932;28:1257-89.

8 Nelson E, Rennels M. Innervation of intracranial arteries. Brain 1970;93:475-90.

9 James IM, Millar RA, Purves MJ. Observations on the extrinsic neural control of Cerebral Blood Flow in the Baboon. Circ Res 1969;25:77-93.

10 Harper AM, Deshmukh VD, Rowan JO, Jennett WB. The influence of sympathetic nervous activity on cerebral blood flow. Arch Neurol 1972;27:1-6.

11 Baumbach GL, Heistad DD. Effects of Sympathetic Stimulation and Changes in Arterial Pressure on Segmental Resistance of Cerebral Vessels in Rabbits and Cats. Circ Res 1983;52:527-33.

12 Wagerle LC, Kumar SP, Delivoria-Papadopoulos M. Effect of Sympathetic Nerve Stimulation on Cerebral Blood Flow in Newborn Piglets. Paediatr Res 1986;20:131-5.

13 Greenfield JC Jr, Tindall GT. Effect of norepinephrine, and angiotensin, on blood flow in the internal carotid artery of man. $J$ Clin Invest 1968;47:1672-84.

14 Toda N, Fujita Y. Responsiveness of isolated cerebral and peripheral arteries to serotonin, norepinephrine, and transmural electrical stimulation. Circ Res 1973;33:98-104.

15 Caronna J, Plum F. Cerebrovascular regulation in preganglionic and postganglionic autonomic insufficiency. Stroke 1973;4: 12-19.

16 Meyer JS, Shimazu K, Fukuuchi Y, et al. Cerebral dysautoregulation in central neurogenic orthostatic hypotension (ShyDrager syndrome). Neurology 1973;23:262-73.

17 Nanda RN, Wyper DJ, Harper AM, Johnson RH. Cerebral blood flow in paraplegia. Paraplegia 1974;12:212-8.

18 Thomas DJ, Bannister R. Preservation of Autoregulation of Cerebral Blood Flow in Autonomic Failure. J Neurol Sci 1980;44:205-12.

19 Aaslid R, Huber P, Nornes H. Evaluation of cerebrovascular spasm with transcranial doppler ultrasound. $J$ Neurosurg 1984;60:37-41.

20 Bishop CCR, Powell S, Rutt D, Browse NL. Transcranial doppler measurement of middle cerebral artery blood flow velocity: a validation study. Stroke 1986;17:913-5.

21 Barnett AJ, Wagner GR. Severe orthostatic hypotension: case report and description of response to sympathomimetic drugs. Am Heart J 1958;56:412-24.

22 Parks VJ, Sandison AG, Skinner SL, Whelan RF. Sympathomimetic drugs in orthostatic hypotension. Lancet 1961;i: 1133-6.

23 Thomas DJ, Zilkha E, Redmond S, du Boulay GH, et al. An intravenous ${ }^{133}$ Xenon clearance technique for measuring cerebral blood flow. J Neurol Sci 1979;40:53-63.

24 Heistad DD, Marcus ML, Gross PM. Effects of sympathetic nerves on cerebral vessels in dog, cat and monkey. Am J Physiol 1978;235:H544-H552.

25 Davies B, Bannister R, Sever P. Pressor amines and monoamineonidase inhibitors for treatment of postural hypotension in autonomic failure. Lancet 1978;i:172-5.

26 Obrist WD, Wilkinson WE. The non-invasive Xe-133 method: Evaluation of CBF indices. In: (Bes A, Geraud G eds.), Cerebral Circulation Amsterdam, Elsevier, N. Holland Inc., 1980: 119-24.

27 Mathias CJ, Bannister R, Raimbach SJ, Cortelli P. Clinical, neurochemical, and neuropharmacological findings in 2 siblings with sympathetic failure due to dopamine- $\beta$-hydroxylase deficiency, before and after treatment with D-L-3-hydroxyphenylserine. J Neurology 1988;235:S74. 